\title{
Erratum to: Comparative transcriptome analysis of early somatic embryo formation and seed development in Brazilian pine, Araucaria angustifolia (Bertol.) Kuntze
}

Paula Elbl • Roberta Alvares Campos • Bruno Silvestre Lira - Sonia Cristina Silva Andrade • Leonardo Jo • Andre Luis Wendt dos Santos • Luiz Lehmann Coutinho •

Eny Iochevet Segal Floh • Magdalena Rossi

Published online: 4 February 2015

(C) Springer Science+Business Media Dordrecht 2015

\section{Erratum to: Plant Cell Tiss Organ Cult}

DOI 10.1007/s11240-014-0523-3

The list of authors in the original publication was incomplete. It should have included author Roberta Alvares Campos, as listed in this erratum.

The online version of the original article can be found under doi:10. 1007/s11240-014-0523-3.

P. Elbl · R. A. Campos - B. S. Lira - L. Jo ·

A. L. W. dos Santos · E. I. S. Floh $(\bowtie) \cdot$ M. Rossi $(\bowtie)$

Departamento de Botânica, Instituto de Biociências,

Universidade de São Paulo, Rua do Matão, 277, São Paulo,

São Paulo 05508-900, Brazil

e-mail: enyfloh@usp.br

M. Rossi

e-mail: mmrossi@usp.br

P. Elbl

e-mail: paulaelbl@gmail.com

R. A. Campos

e-mail: betacamp2003@gmail.com

B. S. Lira

e-mail: bslira.bsl@gmail.com
L. Jo

e-mail: jo.leonardo85@gmail.com

A. L. W. dos Santos

e-mail: alwsantos@yahoo.com.br

S. C. S. Andrade · L. L. Coutinho

Departmento de Zootecnia, ESALQ, Universidade de São Paulo,

Av. Pádua Dias, 11, Piracicaba, São Paulo 13419-900, Brazil

e-mail: soniacsandrade@gmail.com

L. L. Coutinho

e-mail: 1lcoutinho@usp.br 